\title{
Caracterización alofónica del grupo fonémico /tr / en el habla no-estándar de Chile en situación de lectura en voz alta y de entrevista semiespontánea
}

\author{
Alejandra Araneda Beltrán ${ }^{1}$ \\ Universidad de Concepción, Chile \\ Jaime Soto-Barba \\ Universidad de Concepción, Chile \\ Daniel Pereira Pereira \\ Universidad de Concepción, Chile \\ Mauricio A. Figueroa Candia \\ Universidad de Concepción, Chile
}

\section{Resumen}

En este artículo se presentan los principales resultados de la variación alofónica del grupo consonántico /tr// en el español no-estándar chileno de ocho ciudades del país (Iquique, La Serena, Valparaíso, Santiago, Concepción, Temuco, Coyhaique y Punta Arenas), en situación de entrevista semiespontánea y de lectura en voz alta, para hablantes de sexo masculino y femenino. Los resultados muestran que la mayor cantidad de fonos que realiza el fonema $/ \mathrm{trc} /$ corresponde

\footnotetext{
Para correspondencia, dirigirse a: Alejandra Araneda Beltrán (alaraneda@udec.cl), Jaime Soto-Barba (jstot@udec.cl), Daniel Pereira Pereira (danielpereira@udec.cl) o Mauricio A. Figueroa Candia (maufigueroa@udec.cl), Universidad de Concepción, Departamento de Español, Casilla 160-C. Correo 3, Concepción, Chile.
} 
al sonido oclusivo seguido de vibrante simple [tr]. Además, las variables zona geográfica, modalidad discursiva y sexo influyen de manera estadísticamente significativa en las realizaciones del grupo consonántico en estudio.

Palabras clave: variación alofónica, español no-estándar, entrevista semiespontánea, lectura en voz alta, sonido oclusivo, vibrante simple.

AllophONIC CHARACTERIZATION OF THE PHONEMIC GROUP / Tri / IN NON-STANDARD SPEECH IN A SAMPLE OF CHILEAN SPANISH SPEAKERS READING-ALOUD AND IN A SEMI-SPONTANEOUS INTERVIEW

\section{Abstract}

This paper presents the main results of the allophonic variation of the consonantal group $/ \mathrm{tr} /$ in a variety of Chilean Spanish -that of speakers that have not completed secondary education- obtained from eight cities (Iquique, La Serena, Valparaíso, Santiago, Concepción, Temuco, Coyhaique and Punta Arenas), using two elicitation tasks -semi-spontaneous interview and reading-aloud- completed by male and female speakers. The results showed that the sequence of plosive followed by a tap, that is $/ \mathrm{tr} /$, was more frequent. In addition, the independent variables geographic origin, elicitation task and gender were shown to have a statistically significant effect on the phonetic realizations of $/ \mathrm{tr} /$.

Keywords: allophonic variation, non-standard Spanish, semispontaneous interview, reading-aloud, plosives, tap.

Recibido: 26/01/19 Aceptado: 30/09/19

\section{INTRODUCCIÓN ${ }^{2}$}

El presente estudio tiene por objetivo caracterizar la variación alofónica del grupo consonántico /trr/ en el español no-estándar chileno de ocho ciudades

2 Investigación realizada en el contexto del proyecto Fondecyt 1161466 denominado "Realizaciones fónicas segmentales en habla subestándar: una contribución a la construcción de un inventario inclusivo y actualizado de fonemas y alófonos del español de Chile".

Estudios de postgrado financiados por CONICYT-PFCHA/Magíster Nacional/2018-folio 22170539 . 
del país, en situación de entrevista semiespontánea y de lectura en voz alta, en cuanto a su procedencia geográfica, el género de los informantes y la modalidad discursiva. Como es conocido, los precedentes de investigación sobre el grupo consonántico /trc/son abundantes y diversos. Con la finalidad de no reiterar en detalle asuntos que ya han sido tratados de manera monográfica en otros lugares, proveeremos una síntesis del estado de la cuestión en los siguientes párrafos (para trabajos que desarrollan in extenso estas temáticas, véase Figueroa 2008).

a. El estudio del grupo consonántico /tr $/$ y los fonos que lo realizan presenta bastante material bibliográfico, en el que confluyen diversas perspectivas teóricas que coinciden en afirmar la dispersión del fenómeno de las variantes de /trr/ en términos geográficos. Debido a que las variantes del grupo consonántico se han expandido por toda España (Menéndez Pidal 1962; Navarro Tomás 1918) y por varios países de América (Canfield 1962; Gaínza 1976; Fontanella 1992), es decir, están presentes en todos los países hispanohablantes (Quilis 1999; Amado Alonso 1953).

b. Los autores señalan que el grupo consonántico $/ \mathrm{tr} /$ presenta tres variantes: Pidal 1962; Navarro Tomás 1918; Quilis 1999; Canfield 1962; Gaínza 1976; Fontanella 1992; Lenz 1892; Silva-Fuenzalida 1952-1953; Oroz 1966; Véliz, Araya y Rodríguez 1977; Cepeda 1991; Contreras 1993; Salas 1996-1997; Rabanales 2000; Figueroa, Soto-Barba y Ñanculeo, 2010; Figueroa, Salamanca y Nanculeo, 2013). Una variante formada por una oclusiva más una vibrante simple, transcrita habitualmente como [trc], algunos de esos autores señalan su mayor prestigio y asociación a la clase social alta; una segunda variante africada con gran tendencia a la afonía y asibilación (asimilación), usualmente transcrita como [t $\left.\mathrm{t}^{\mathrm{t}}\right]$, que presenta menor prestigio y está asociada al nivel sociocultural medio-bajo -según algunos de estos autores (Amado Alonso 1953); y una tercera variante fricativa, transcrita como [İ], que presenta estigmatización y se asocia al nivel sociocultural bajo de acuerdo con algunos autores. Por lo anterior en esta muestra de español no-estándar se espera encontrar mayores porcentajes de realización de los fonos fricativos y africados.

c. Los distintos grados de prestigio y estigmatización de las variantes de /trc/ están asociadas a la valoración subjetiva que realizan los hablantes de cada una de ellas (Valdivieso 1978-1988; Felix Bobadilla y Gustavo Bobadilla 1980-1981; Tassara 1993-1994) y al manejo de un código restringido o elaborado por parte de los informantes (Wigdorsky 19781983). A pesar de la asociación de cada variante a un nivel sociocultural y a distintos grados de prestigio y estigmatización, varios autores señalan que la estigmatización de variantes africadas y fricativas ha disminuido a 
lo largo del tiempo por su extensión a distintas modalidades de habla y a diferentes estratos socioculturales (Espinosa y Contardo 1992; Rabanales 1992; Salas 1996-1997).

d. El fenómeno de asibilación de las variantes en un principio estaba limitado al habla inculta informal o al habla popular, pero con el paso del tiempo este proceso se expandió a los otros estratos socioculturales, generando mayor aceptación por parte de los hablantes (Rabanales 2000). Por una parte, el género de los hablantes es una variable que podría influir en las realizaciones del grupo consonántico en estudio, pues las mujeres prefieren la variante estándar en mayor proporción a los hombres cuando se les compara (Cepeda 1991; Tassara 1993-1994). Por otra parte, las variables de contexto fonético y frecuencia de la palabra no inciden en las realizaciones de $/ \mathrm{tr} /$ (Figueroa, et al. 2010), por lo que son descartadas de la presente investigación.

\section{METODOLOGÍA}

\subsection{TIPO DE INVESTIGACIÓN}

El presente estudio tiene un enfoque cuantitativo, ya que "utiliza la recolección de datos para probar hipótesis con base en la medición numérica y el análisis estadístico, con el fin de establecer pautas de comportamiento y probar teorías" (Hernández, Fernández y Baptista, 2014: 4). Además, este enfoque consta de las siguientes etapas: idea, planteamiento del problema, revisión de la literatura y desarrollo del marco teórico, visualización del alcance del estudio, elaboración de hipótesis y definición de variables, desarrollo del diseño de investigación, definición y selección de la muestra, recolección de los datos, análisis de los datos y elaboración del reporte de resultados. (Hernández et al., 2014: 5). Al mismo tiempo, la presente investigación tiene un alcance descriptivo, porque "busca especificar propiedades y características importantes de cualquier fenómeno que se analice, describe tendencias de un grupo o población" (Hernández et al., 2014: 92).

\subsection{TOMA DE MUESTRAS, TAREAS Y GRABACIONES}

Los informantes de esta investigación corresponden a hablantes de la muestra recopilada para el proyecto Fondecyt 1161466 denominado "Realizaciones 
fónicas segmentales en habla subestándar: una contribución a la construcción de un inventario inclusivo y actualizado de fonemas y alófonos del español de Chile" en curso, cuyo objetivo general es identificar y describir las realizaciones fónicas segmentales del habla no-estándar chilena con el fin último de elaborar un inventario oficial con los sonidos del español en Chile. En el proyecto Fondecyt 1161466, la toma de muestras se llevó a cabo en un período de dos años y medio, en ocho ciudades del país, seleccionadas sobre la base de la propuesta de Wagner (2004): por la zona norte, Iquique y La Serena; por la zona centro, Santiago y Valparaíso; por la zona sur, Concepción y Temuco; y, por la zona sur-austral, Coyhaique y Punta Arenas. El número total de sujetos considerados en esta ocasión ascendió a 160 hablantes.

El proyecto Fondecyt utilizó tres tareas de elicitación de habla que correspondieron a una entrevista semiespontánea en la que el sujeto respondía a preguntas personales y generales sobre sus experiencias de vida; una lectura en voz alta de oraciones diseñadas especialmente para observar aspectos fonéticos-fonológicos de interés para la investigación; y un juego interactivo de descripción de láminas denominado DiapixSp (Figueroa, García y Salamanca, en evaluación). De estas tres tareas, se escogieron dos para la presente investigación; estas son, la entrevista semiespontánea y la lectura en voz alta. En algunos casos muy específicos en que no se contó con el número de ocurrencias mínimas que se propuso para esta investigación, se completó la muestra con instancias elicitadas a través de DiapixSp. El habla de los sujetos fue registrada mediante sistemas de micrófonos de cabeza Sennheiser EW-152-G3, cuya señal fue enviada a grabadoras digitales Tascam DR40. Las grabaciones se realizaron con una frecuencia de muestreo de 44.100 $\mathrm{Hz}$, una profundidad de 24 bits, en modalidad estéreo y formato WAV (el discurso de cada participante se registró en un canal de la señal estéreo).

\subsection{INFORMANTES DE ESTE ESTUDIO}

Para el desarrollo de esta investigación, se seleccionaron veinte informantes de cada ciudad en la modalidad de entrevista semiespontánea y de lectura en voz alta, para ocho ciudades, dando un total de 160 hablantes, escogidos de acuerdo con los siguientes criterios:

1. Hablantes mayores de 20 años.

2. Hablantes con enseñanza media incompleta.

3. Hablantes con nacimiento y permanencia en la ciudad de origen.

4. Hablantes sin anomalías fonoarticulatorias.

5. Hablantes de nacionalidad chilena. 
6. Hablantes sin conocimiento avanzado de otra lengua (por ejemplo, inglés, francés, mapudungun).

7. Hablantes alfabetizados.

Durante la toma de la muestra, se tuvo cuidado de equilibrar la variable de género en cada una de las ciudades; cabe destacar que en las ciudades de Iquique y Concepción fueron las únicas donde no se dispuso de este equilibrio por lo que se debieron dejar ocho hombres y doce mujeres en la primera ciudad, y nueve mujeres y once hombres en la segunda ciudad mencionada.

\subsection{VARIANTES CONSIDERADAS}

Para efectos de la presente investigación, se reconocerán las variantes del grupo consonántico /trc/ presentadas en la Tabla 1, estas variantes alofónicas coinciden con las propuestas por Figueroa, et al. (2010: 20-27).

\begin{tabular}{|c|c|c|}
\hline Número & Variante & Descripción \\
\hline 1. & {$[\mathrm{tc} r]$} & Oclusiva seguida de vibrante simple. \\
\hline 2. & {$\left[\mathrm{t}^{2}\right]$} & Africada asibilada con fricción breve. \\
\hline 3. & {$\left[\begin{array}{lll}\mathrm{t} & 0 \\
\mathrm{~J}_{\mathrm{t}}\end{array}\right]$} & Africada asibilada con fricción larga. \\
\hline 4. & {$\left[\begin{array}{ccc}0 \\
1 \\
1\end{array}\right]$} & Fricativa. \\
\hline
\end{tabular}

Tabla 1. Alófonos del grupo consonántico /trc/ en la muestra

\subsection{ANÁlisis FONÉTICO-AUDITIVO Y FONÉTICO-ACÚSTICO EN MODALIDAD DE ENTREVISTA Y DIAPIX}

El análisis de las ocurrencias de / semiespontánea se realizó como se detalla a continuación. En primer lugar, se identificaron las primeras diez ocurrencias del grupo consonántico /trc/ de cada sujeto, en el caso de no poder completar las diez realizaciones mediante las muestras obtenidas en situación de entrevista se recurrió a los registros de la tarea DiapixSp, lo que ocurrió solamente en un par de ocasiones.

En segundo lugar, se utilizó el método de análisis auditivo clásico contrastado para escuchar y transcribir las emisiones del grupo consonántico que suman un total de 1.600. Durante el proceso de constitución del corpus, investigadores entrenados clasificaron cada una de las realizaciones del grupo $/ \mathrm{tr} /$ en una de las variantes alofónicas definidas en la Tabla 1. Luego, la clasificación de cada uno de ellos fue comparada para llegar a un consenso. 
En tercer lugar, en caso de discrepancias se procedió a realizar un análisis espectrográfico del segmento, a través del programa Praat (Boersma y Weenink: 2018, versión 6.0.39). Sobre la base de lo propuesto por Figueroa et al. (2010: 17) se siguieron los criterios fonético-acústicos de duración del segmento oclusivo, duración del segmento vibrante o fricativo, según sea el caso y la proporción de oclusión opuesta a vibración/fricción para adscribir los casos dudosos a una de las variantes caracterizadas espectrográficamente en dicho trabajo.

\subsection{ANÁLISIS FONÉTICO-AUDITIVO Y FONÉTICO-ACÚSTICO EN MODALIDAD DE LECTURA EN VOZ ALTA}

El análisis de las ocurrencias de /trs/ en la modalidad de lectura en voz alta se realizó con los mismos criterios establecidos para el corpus de entrevista, con la salvedad de que en este caso se seleccionaron dos ocurrencias por cada contexto fonético definido en la lectura de oraciones. Los contextos fonéticos a considerar son: posición inicial absoluta, posición intervocálica, precedido de consonante nasal y precedido de consonante fricativa. De esta forma, en la modalidad de lectura en voz alta se analizaron 1.280 realizaciones, ya que cada hablante aportó con ocho ocurrencias. Si se consideran ambas modalidades se tiene un corpus de habla con 2.880 ocurrencias del grupo consonántico /tr /. Cabe destacar que la variante de contexto fonético se consideró en modalidad de lectura para seleccionar las realizaciones, pero no se analizará en los resultados por no presentar significancia estadística según Figueroa et al. (2010:29-30), reseñado en la introducción.

\section{ANÁLISIS DE RESULTADOS}

En este apartado, se revisarán los resultados obtenidos en la investigación en modalidad de entrevista semiespontánea y de lectura en voz alta, comenzando por las tendencias nacionales, siguiendo por los resultados en cada ciudad. Luego se observará la incidencia del género de los informantes en las realizaciones de $/ \mathrm{t} r / \mathrm{y}$ se establecerá una comparación entre las dos modalidades discursivas incluidas en este estudio. Además de la observación de tendencias, se reportará hacia el final de esta sección un análisis estadístico que evaluó la incidencia de las variables independientes zona geográfica, 
sexo y modalidad discursiva en la expresión de las variantes del grupo consonántico en estudio.

\subsection{ANÁlisis DE RESUlTAdos EN MODALIDAD DE ENTREVISTA}

\subsubsection{Resultados generales según alófonos de /trr/ en entrevista}

\begin{tabular}{|c|c|c|}
\hline Variante fonética & Realizaciones & Porcentaje \\
\hline$\left[\begin{array}{ll}\mathrm{t} r \\
\mathrm{n}\end{array}\right]$ & 1282 & $80,1 \%$ \\
\hline$\left[\mathrm{t}^{i}\right]$ & 280 & $17,5 \%$ \\
\hline$\left[{ }^{\mathrm{t}} \mathrm{I}_{\mathrm{L}}^{0}\right]$ & 36 & $2,3 \%$ \\
\hline$\left[\begin{array}{ll}0 \\
1 \\
1\end{array}\right]$ & 2 & $0,1 \%$ \\
\hline Totales & 1600 & $100 \%$ \\
\hline
\end{tabular}

Tabla 2. Resultados generales en entrevista, según alófonos de $/ \underset{n}{\mathrm{tr}} /$

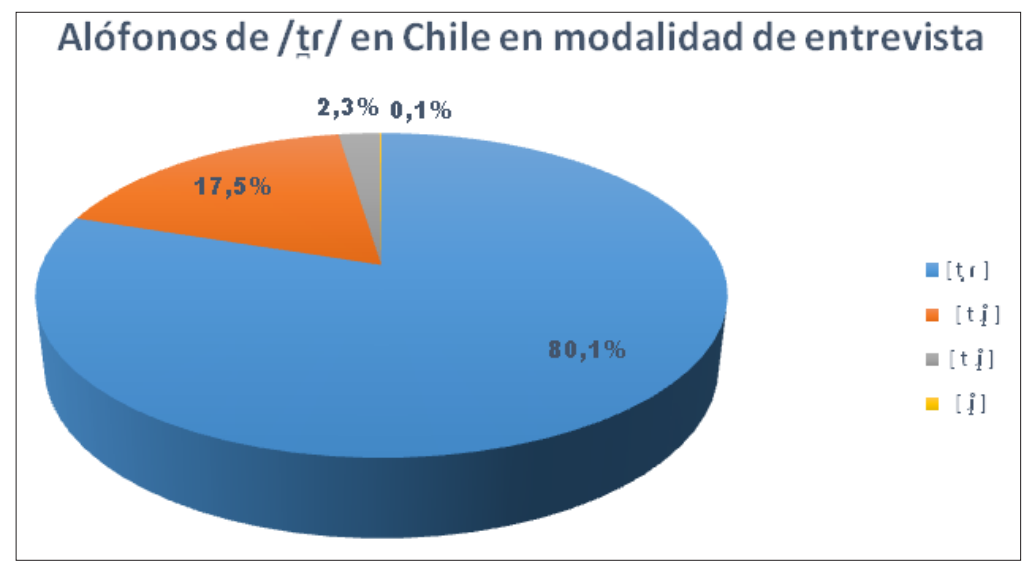

Figura 1. Gráfico de resultados generales en entrevista, según alófonos de /tr $/$

Dentro de los resultados generales de la distribución de los alófonos del grupo consonántico / trr/a lo largo de las ocho ciudades estudiadas en modalidad de entrevista, se aprecia en la Tabla 2, y la Figura 1 que la variante oclusiva seguida de vibrante simple [trc] es la mayor realizada dentro de la muestra evaluada con un $80,1 \%$, le sigue la variante africada asibilada con fricción breve [t] con un $17,5 \%$ y la africada asibilada con fricción larga [t.j] con un $2,3 \%$. La variante fricativa [⿺辶⿸广巳]. 
muestra, lo que equivale a dos realizaciones que aparecieron en las ciudades de Santiago y Valparaíso.

\subsubsection{Análisis comparativo entre las ciudades del país en modalidad de entrevista}

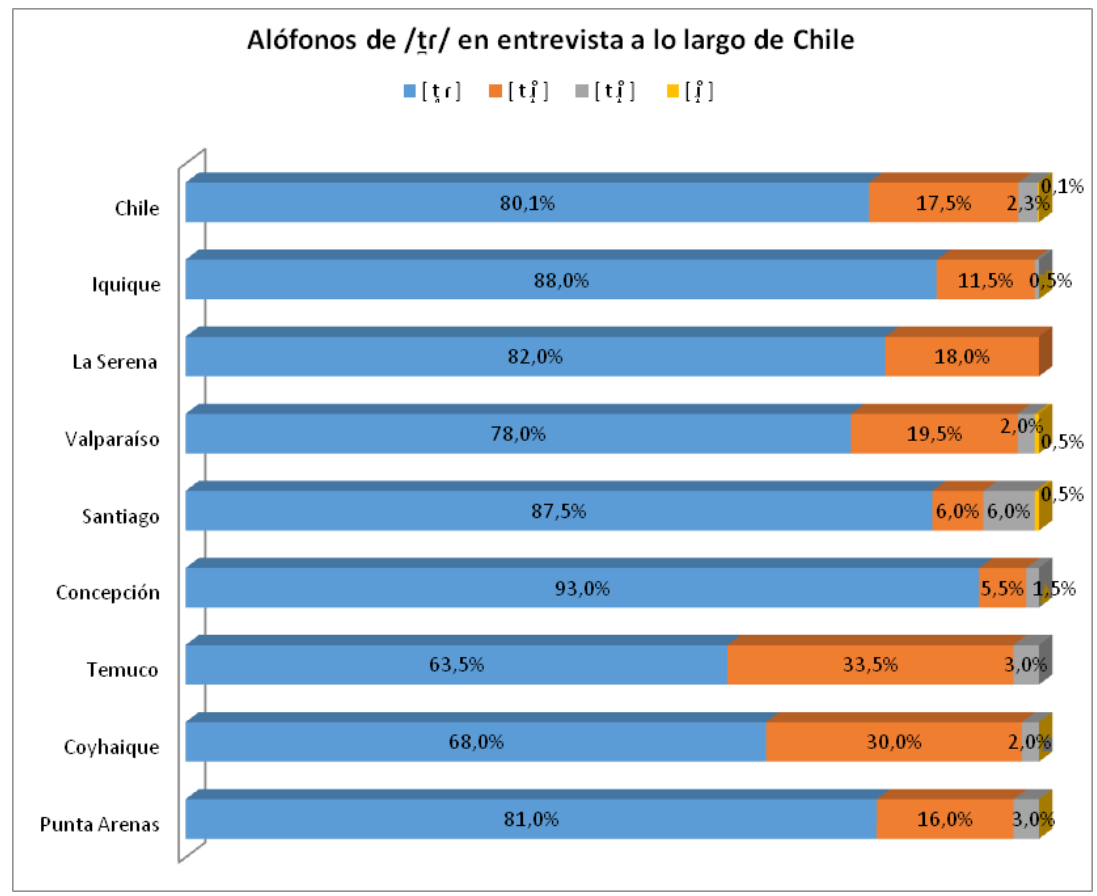

Figura 2. Gráfico de los resultados de las variantes de /trs/ en todas las ciudades del país en modalidad de entrevista

En la ciudad de Iquique, como se observa en la Figura 2, la distribución alofónica de /trr/ estuvo marcada por la preponderancia de la variante oclusiva seguida de vibrante simple [tr] con un $88 \%$, seguida por la africada asibilada con fricción breve [t] con un $11,5 \%$ y, finalmente se encuentra la africada asibilada con fricción larga [t.̃.] con un $0,5 \%$. Además, en esta ciudad, no se presenta ninguna realización de la variante fricativa [i̊l] $]$. En La Serena, se evidencia la preferencia por el alófono oclusivo seguido de vibrante simple [trc] con un $82 \%$ de las ocurrencias, lo sigue la variante africada asibilada con fricción breve [t. 
no se presentan ocurrencias del fono africado asibilado con fricción larga [ț.] ni del fono fricativo [i̊n]

En la ciudad de Valparaíso, como muestra la Figura 2, la distribución alofónica del grupo consonántico/tr/ se caracteriza por evidenciar preferencia por la variante oclusiva seguida de vibrante simple [tr ] que posee un $78 \%$ de las realizaciones, la sigue la africada asibilada con fricción breve [t $[\mathrm{t}]$ con un $19,5 \%$, la africada asibilada con fricción larga [t] $\mathrm{t}]$ con un $2 \%$ y la variante

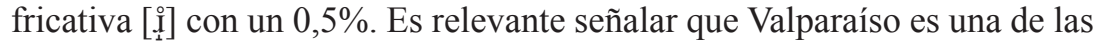
dos ciudades donde se evidencian los cuatro alófonos en estudio, a diferencia del resto de las ciudades donde solo se observan dos o tres de ellos. En la ciudad de Santiago, se observa en la Figura 2, una notoria preferencia por la variante oclusiva seguida de vibrante simple [trc] con un $87,5 \%$, le sigue la variante africada asibilada con fricción breve [t] con un $6 \%$ de las realizaciones, luego está la africada asibilada con fricción larga [t.J] con un $6 \%$ y la variante fricativa $\left[\begin{array}{l}1 \\ 1\end{array}\right]$ con un $0,5 \%$. Cabe destacar que en esta ciudad la segunda y tercera variante de preferencia poseen el mismo porcentaje con doce ocurrencias cada una. Además, es relevante señalar que Santiago, al igual que Valparaíso, es la segunda ciudad que presenta los cuatro alófonos en estudio, a diferencia del resto de las ciudades donde solo se observan dos o tres de ellos. Es destacable también que en Santiago se encuentre el mayor porcentaje de ocurrencias de la tercera variante, esto es, [t.j].

En la ciudad de Concepción, tal como lo muestra la Figura 2, se observa preferencia por la variante oclusiva seguida de vibrante simple [trc] con un $93 \%$, le sigue la variante africada asibilada con fricción breve [t] con un $5,5 \%$ de las realizaciones, y finalmente está la africada asibilada con fricción larga [t.'.] con un 1,5\%; cabe señalar que en esta ciudad no se presenta ninguna realización de la variante fricativa [̊̊̊] $]$, fenómeno que coincide con lo observado en Iquique y La Serena. Además, es destacable que en Concepción se registre el mayor porcentaje de realizaciones de la primera variante, es decir, [tr $]$; también se constata el menor porcentaje entre todas las ciudades del segundo fono, o sea, la africada asibilada con fricción breve. En la ciudad de Temuco, se observa preferencia por la variante oclusiva seguida de vibrante simple $[\mathrm{t} r]$ con un $63,5 \%$, le sigue la variante africada asibilada con fricción breve [t'] con un 33,5\% de las realizaciones, y finalmente está la africada asibilada con fricción larga ['̛̃] con un 3\%; también en esta ciudad no se presenta ninguna realización de la variante fricativa [̊̊̊ 1$]$, como también sucede en Iquique, La Serena y Concepción. Además, en Temuco, se registra el menor porcentaje de ocurrencias del primer fono, vale decir, [tr $]$; adicionalmente, se constata el mayor porcentaje entre todas las ciudades del segundo fono, es decir, [t. 
En Coyhaique, se observa en la Figura 2, preferencia por la variante oclusiva seguida de vibrante simple [tr] con un $68 \%$, le sigue la variante africada asibilada con fricción breve [t] con un $30 \%$ de las realizaciones $\mathrm{y}$, finalmente, se observa la africada asibilada con fricción larga [t.̃.] con un $2 \%$; además, en esta ciudad, no se presenta ninguna realización de la variante fricativa [ $\left.\begin{array}{c}0 \\ 1\end{array}\right]$, lo que se asemeja al comportamiento de Iquique, $\mathrm{La}$ Serena, Concepción y Temuco. En la última ciudad, Punta Arenas, existe mayor porcentaje de ocurrencias de la variante oclusiva seguida de vibrante simple [tr] con un $81 \%$, le sigue la variante africada asibilada con fricción breve $[t]$ con un $16 \%$ de las realizaciones y, finalmente, se aprecia la africada asibilada con fricción larga [t..] con un 3\%; también en esta ciudad, no se

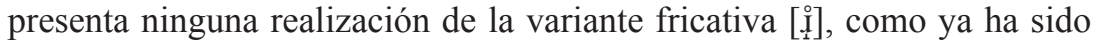
una constante que se manifestó en las ciudades de Iquique, La Serena, Concepción, Temuco y Coyhaique.

Al realizar un análisis comparativo del comportamiento alofónico de /tr $/$ en las ocho ciudades del país en modalidad de entrevista, se constata que en todas las ciudades consideradas, las variantes con mayor porcentaje de realización, en orden decreciente, fueron: el fono oclusivo seguido de vibrante simple [tr $]$, entre un $93 \%$ en Concepción y un $63,5 \%$ en Temuco; el fono africado asibilado con fricción breve [t], entre un 33,5\% en Temuco y un 5,5\% en Concepción; y el fono africado asibilado con fricción larga [t] $\mathrm{t}$ ], entre un $6 \%$ en Santiago y un $0 \%$ en La Serena. Además, el fono fricativo

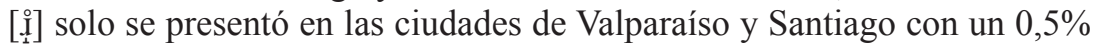
en cada una de ellas. Por lo anterior, todas las ciudades observadas repiten la tendencia nacional en términos de sus primeros tres fonos de preferencia y de su orden jerárquico, a excepción de La Serena que solo presenta los dos primeros fonos, por lo que el comportamiento alofónico nacional tiende a presentar homogeneidad.

En términos de la distribución porcentual entre los fonos en estudio, las ciudades se pueden reunir en tres grupos: el primero considera a Concepción (93\%), Iquique (88\%) y Santiago $(87,5 \%)$ quienes presentan los mayores porcentajes en la primera variante según el orden nacional; luego, en el segundo grupo, se encuentran La Serena (82\%), Punta Arenas (81\%) y Valparaíso (78\%) con porcentajes intermedios en la primera variante; finalmente en el tercer grupo están Coyhaique (68\%) y Temuco $(63,5 \%)$ con los menores porcentajes de la primera variante de preferencia nacional. 
3.1.3. Resultados generales según género de los informantes en entrevista

\begin{tabular}{|c|c|c|c|c|}
\hline \multirow[b]{2}{*}{$\begin{array}{l}\text { Variante } \\
\text { fonética }\end{array}$} & \multicolumn{2}{|c|}{ Mujer } & \multicolumn{2}{|c|}{ Hombre } \\
\hline & $\begin{array}{l}\text { Número de } \\
\text { realizaciones }\end{array}$ & Porcentaje & $\begin{array}{l}\text { Número de } \\
\text { realizaciones }\end{array}$ & Porcentaje \\
\hline$[\underset{n}{t} \mathrm{r}]$ & 722 & $87 \%$ & 560 & $72,7 \%$ \\
\hline$\left[\mathrm{t}^{i}\right]$ & 107 & $12,9 \%$ & 173 & $22,5 \%$ \\
\hline$\left[\begin{array}{ll}\mathrm{t} & 0 \\
{ }^{1}\end{array}\right]$ & 1 & $0,1 \%$ & 35 & $4,5 \%$ \\
\hline [욤] & 0 & $0 \%$ & 2 & $0,3 \%$ \\
\hline Totales & 830 & $100 \%$ & 770 & $100 \%$ \\
\hline
\end{tabular}

Tabla 3. Resultados generales de las variantes de /tr / según género de los informantes en modalidad de entrevista

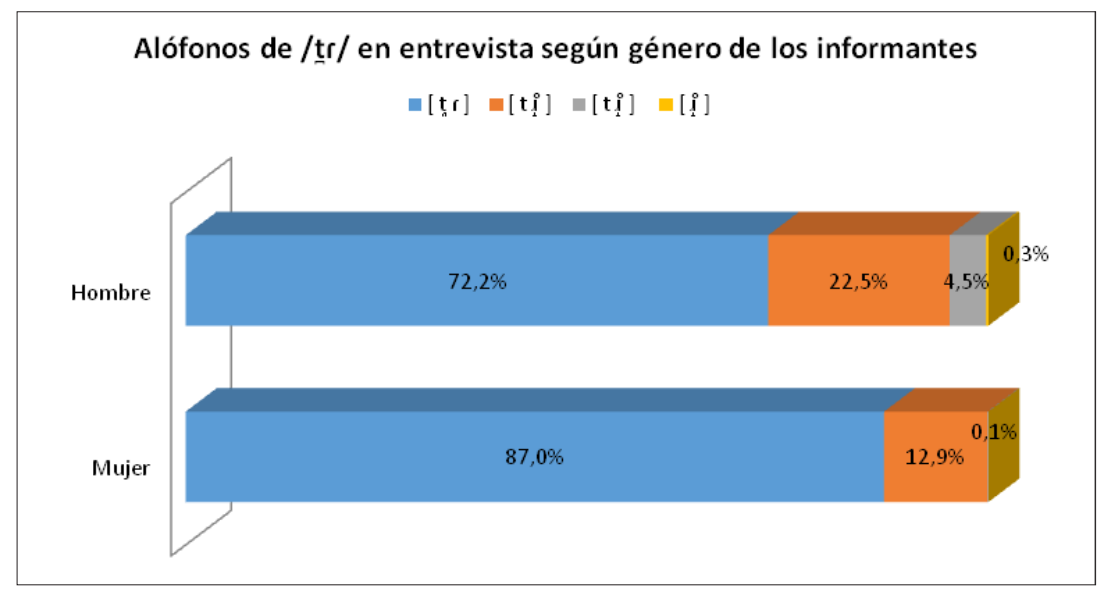

Figura 3. Gráfico de resultados generales de las variantes de / $\mathrm{tr} /$ según género de los informantes en modalidad de entrevista

En la Tabla 3 y la Figura 3, al analizar el comportamiento alofónico del grupo consonántico /trr/a nivel nacional, según el género de los informantes en modalidad de entrevista, en primer lugar, se observa que los hombres y las mujeres realizan con mayor frecuencia las variantes oclusiva seguida de vibrante simple $[\mathrm{tr}]$ y la africada asibilada con fricción breve [t $[\mathrm{t}$ ], cuyos porcentajes de ocurrencia son: $72,2 \%$ y $22,5 \%$ en el caso de los hombres; y $87 \%$ y $12,9 \%$ en el caso de las mujeres. Por lo tanto, poseen comportamientos bastante similares en la jerarquía otorgada a los dos primeros alófonos, pero 
sus porcentajes son diferentes, ya que las mujeres realizan más oclusivas seguidas de vibrantes simples y menos africadas asibiladas con fricción breve en comparación con las realizaciones de los hombres. En segundo lugar, al analizar las realizaciones de la variante africada asibilada con fricción larga [t.J], se constata que en el caso de los hombres su porcentaje de realizaciones asciende a un 4,5\% y en el caso de las mujeres a un $0,1 \%$. Además, al observar las realizaciones de la variante fricativa [i̊l] , se constata que los hombres presentan un $0,3 \%$ de ocurrencias y las mujeres no tienen realizaciones de este alófono. Por todo lo anterior, se puede afirmar que el comportamiento alofónico entre hombres y mujeres es similar en términos de la jerarquización de las variantes, pero difiere en términos de la relevancia porcentual que se le otorga a cada alófono.

3.2. AnÁlisis de RESUltados EN MODALIDAD DE LECTURA EN VOZ ALTA

\subsubsection{Resultados generales según alófonos de /trr/ en lectura en voz alta}

\begin{tabular}{|c|c|c|}
\hline Variante fonética & Realizaciones & Porcentaje \\
\hline$\left[\begin{array}{c}\mathrm{t} c \\
\mathrm{c}\end{array}\right]$ & 1174 & $91,7 \%$ \\
\hline$\left[\mathrm{t}^{\mathrm{j}}\right]$ & 91 & $7,1 \%$ \\
\hline$\left[\begin{array}{c}\mathrm{t} \\
\mathrm{I}_{1}^{\circ}\end{array}\right]$ & 14 & $1,1 \%$ \\
\hline$\left[\begin{array}{l}0 \\
1\end{array}\right]$ & 1 & $0,1 \%$ \\
\hline Totales & 1280 & $100 \%$ \\
\hline
\end{tabular}

Tabla 4. Resultados generales en lectura en voz alta, según alófonos de /trr/.

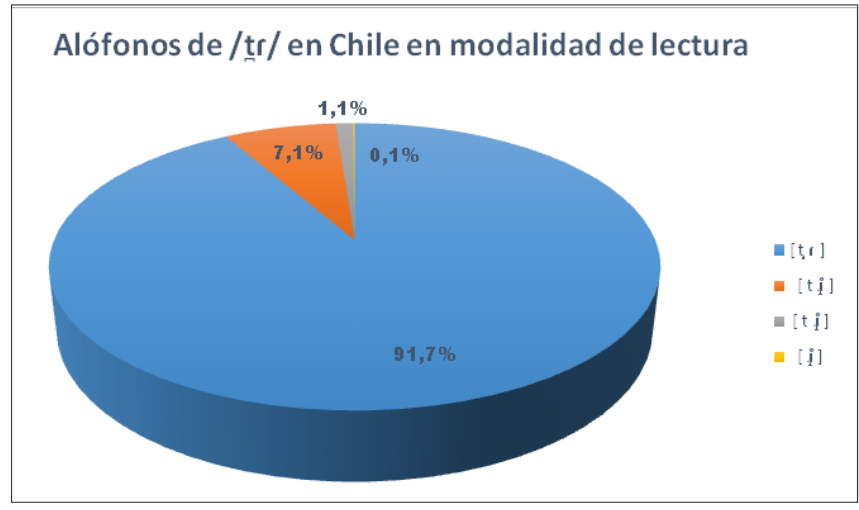

Figura 4. Gráfico de resultados generales en lectura en voz alta, según alófonos de /trc/ 
Dentro de los resultados generales de la distribución de los alófonos del grupo consonántico /trr /, a lo largo de las ocho ciudades estudiadas en modalidad de lectura en voz alta, se aprecia en la Tabla 4 y en la Figura 4 que la variante oclusiva seguida de vibrante simple [tr ] es la mayor realizada dentro de la muestra evaluada con un $91,7 \%$, la sigue la variante africada asibilada con fricción breve [t] con un 7,1\% y la africada asibilada con fricción larga [t.t] con un $1,1 \%$. La variante fricativa [i̊ en toda la muestra, lo que equivale a una realización que se manifestó en Temuco. Estas tendencias son muy similares a las revisadas en la Tabla 2, Figura 1 en modalidad de entrevista, en términos del orden jerárquico de las variantes y de las preferencias registradas por los hablantes, pero se diferencian en los porcentajes que le corresponde a cada una de las variantes.

\subsubsection{Análisis comparativo entre las ciudades del país en modalidad de} lectura en voz alta

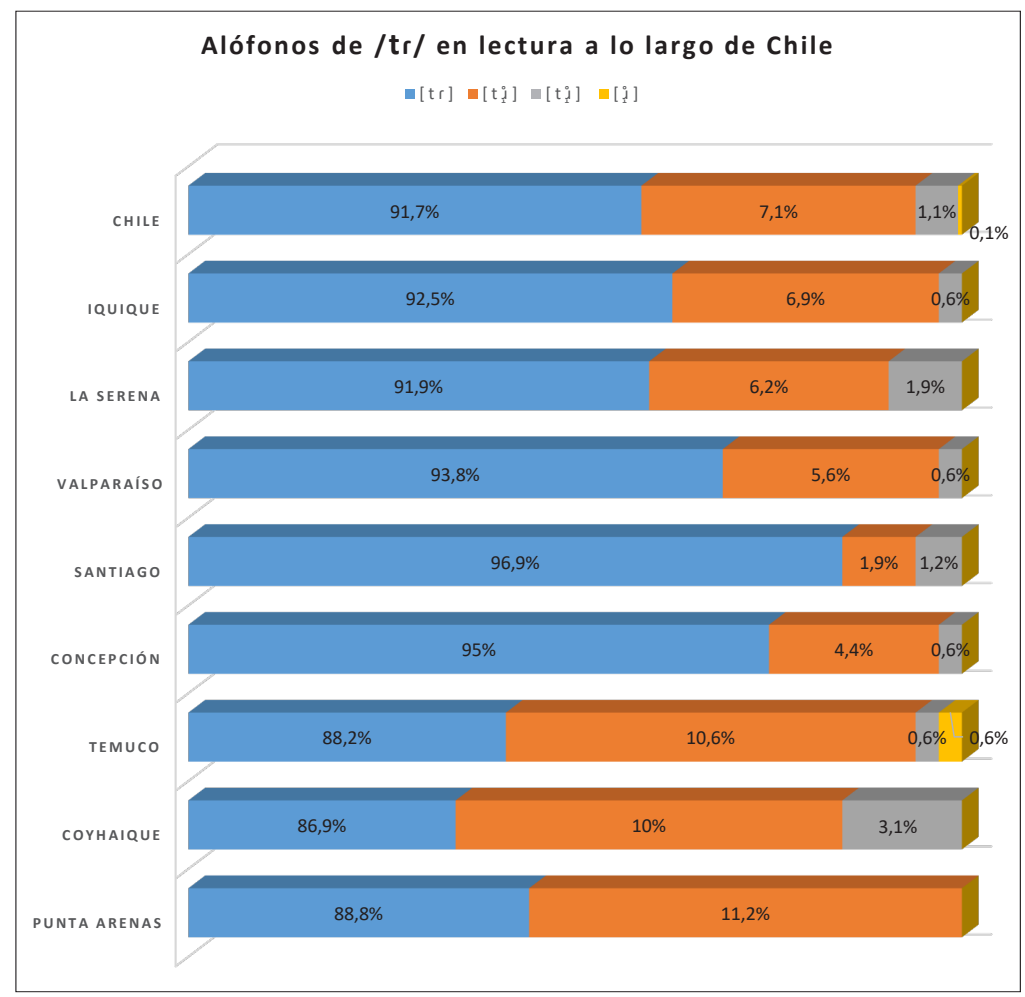

Figura 5. Gráfico de los resultados de las variantes de /trc/ en todas las ciudades del país en modalidad de lectura 
En la ciudad de Iquique, como se observa en la Figura 5, la distribución alofónica de /tr $/$ estuvo marcada por la preponderancia de la variante oclusiva seguida de vibrante simple [tr] con un $92,5 \%$, seguida por la africada asibilada con fricción breve [t] con un $6,9 \% \mathrm{y}$, finalmente se encuentra la africada asibilada con fricción larga [t].] con un $0,6 \%$. Además, en esta ciudad

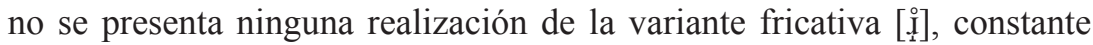
que se replica en la mayoría de las ciudades a excepción de Temuco. En La Serena se evidencia la preferencia por el alófono oclusivo seguido de vibrante simple [tr $]$ con un $91,9 \%$ de las ocurrencias, lo sigue la variante africada asibilada con fricción breve [t] con un $6,2 \%$ y la variante africada asibilada con fricción larga [t’̃.] con un 1,9\%; además no se presentan ocurrencias del fono fricativo [i̊nd] como en la mayoría de las ciudades en modalidad de lectura.

De acuerdo con la Figura 5, en la ciudad de Valparaíso la distribución alofónica del grupo consonántico /trr / se caracteriza por presentar preferencia por la variante oclusiva seguida de vibrante simple [trc] que posee un $93,8 \%$ de las realizaciones, la sigue la africada asibilada con fricción breve [t] con un 5,6\% y la africada asibilada con fricción larga [t.J] con un $0,6 \%$, además no

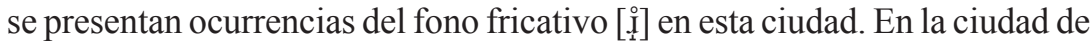
Santiago, se observa en la Figura 5, una notoria preferencia por la variante oclusiva seguida de vibrante simple [trc] con un $96,9 \%$, le sigue la variante africada asibilada con fricción breve $\left[\mathrm{t}^{\mathrm{t}}\right]$ con un $1,9 \%$ de las realizaciones, luego está la africada asibilada con fricción larga [t] ] con un 1,2\% y la variante

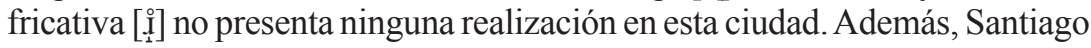
es la ciudad que posee mayor cantidad de realizaciones del fono oclusivo seguido de vibrante simple, también tiene el menor porcentaje entre todas las ciudades el fono africado asibilado con fricción breve.

En la ciudad de Concepción, tal como lo muestra la Figura 5, se observa preferencia por la variante oclusiva seguida de vibrante simple [tr $]$ con un $95 \%$, le sigue la variante africada asibilada con fricción breve [t. de las realizaciones, y finalmente está la africada asibilada con fricción larga [t.̃.] con un $0,6 \%$; además en esta ciudad no se presenta ninguna realización de la variante fricativa [i̊l] $]$. En la ciudad de Temuco se observa en la Figura 5 , preferencia por la variante oclusiva seguida de vibrante simple [tr ] con un $88,2 \%$, le sigue la variante africada asibilada con fricción breve [t] con un $10,6 \%$ de las realizaciones, luego está la africada asibilada con fricción larga [ $\left[\begin{array}{l}\mathrm{t} \\ \mathrm{I}\end{array}\right]$ con un $0,6 \%$ y la variante fricativa $\left[\begin{array}{l}0 \\ 1\end{array}\right]$ con el mismo porcentaje de realizaciones. Es relevante señalar que Temuco es la única ciudad, en modalidad de lectura en voz alta, donde se presentan las cuatro variantes alofónicas del grupo consonántico en estudio, lo que produce mayor variación alofónica en esta ciudad. 
En Coyhaique se observa preferencia por la variante oclusiva seguida de vibrante simple [tr $]$ con un $86,9 \%$, le sigue la variante africada asibilada con fricción breve [t] con un $10 \%$ de las realizaciones, y finalmente está la africada asibilada con fricción larga [t]̃] con un 3,1\%; cabe señalar que en esta ciudad no se presenta ninguna realización de la variante fricativa [:ํ. $]$, lo que se asemeja al comportamiento de la mayoría de las ciudades. Además, esta ciudad es la que posee el menor porcentaje de ocurrencia de [tr ] , también presenta el mayor porcentaje del fono [t.̃.]. En la última ciudad, Punta Arenas, existe mayor porcentaje de ocurrencias de la variante oclusiva seguida de vibrante simple [tr ] con un $88,8 \%$ y le sigue la variante africada asibilada con fricción breve [t? con un $11,2 \%$, el comportamiento de esta ciudad se diferencia de las otras porque no se constata la variante africada

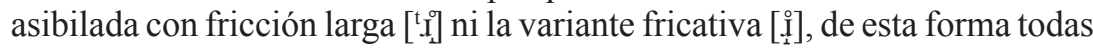
las realizaciones recaen en los dos primeros fonos de preferencia nacional, esto es $[t r]$ y [t]. Además, esta ciudad es la que presenta el mayor porcentaje de realizaciones del segundo fono de preferencia, es decir, [t] y el menor porcentaje del tercer fono, esto es [t.j], entre todas las ciudades.

Al realizar un análisis comparativo del comportamiento alofónico de /tr $/$ entre las ocho ciudades del país en modalidad de lectura en voz alta, se constata que en todas las ciudades consideradas, las variantes con mayor porcentaje de realización, en orden decreciente, fueron: el fono oclusivo seguido de vibrante simple [tr] , entre un $96,9 \%$ en Santiago y un $86,9 \%$ en Coyhaique; el fono africado asibilado con fricción breve [t] , entre un $11,2 \%$ en Punta Arenas y un 1,9\% en Santiago; y el fono africado asibilado con fricción larga [tợ, entre un 3,1\% en Coyhaique y un $0 \%$ en Punta Arenas,

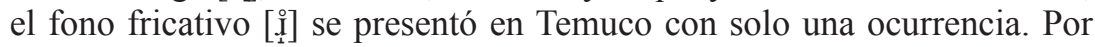
lo mencionado anteriormente, todas las ciudades observadas repiten la tendencia nacional en términos de sus primeros tres fonos de preferencia y de su orden jerárquico, a excepción de Punta Arenas que solo presenta los dos primeros fonos, por lo que el comportamiento alofónico nacional tiende a presentar homogeneidad, tal como se observó en la modalidad discursiva de entrevista.

En términos de la distribución porcentual entre los fonos en estudio, las ciudades se pueden reunir en tres grupos: el primero considera a Santiago (96,9\%), Concepción (95\%), quienes presentan los mayores porcentajes en la primera variante según el orden nacional; luego en el segundo grupo están Valparaíso $(93,8 \%)$, Iquique $(92,5 \%)$, La Serena $(91,9 \%)$ con porcentajes intermedios en la primera variante; finalmente en el tercer grupo están Punta Arenas $(88,8 \%)$, Temuco $(88,2 \%)$ y Coyhaique $(86,9 \%)$ con los menores porcentajes de la primera variante de preferencia nacional. 
3.2.3. Resultados generales según género de los informantes en lectura en voz alta

\begin{tabular}{|c|c|c|c|c|}
\hline & \multicolumn{2}{|c|}{ Mujer } & \multicolumn{2}{|c|}{ Hombre } \\
\hline $\begin{array}{l}\text { Variante } \\
\text { fonética }\end{array}$ & $\begin{array}{l}\text { Número de } \\
\text { realizaciones }\end{array}$ & Porcentaje & $\begin{array}{l}\text { Número de } \\
\text { realizaciones }\end{array}$ & Porcentaje \\
\hline$[\mathrm{t} r]$ & 627 & $94,4 \%$ & 547 & $88,8 \%$ \\
\hline$\left[\mathrm{t}^{1}\right]$ & 30 & $4,5 \%$ & 61 & $9,9 \%$ \\
\hline$\left[{ }^{t}{ }^{t} I_{1}^{0}\right]$ & 7 & $1,1 \%$ & 7 & $1,1 \%$ \\
\hline 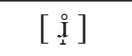 & 0 & $0 \%$ & 1 & $0,2 \%$ \\
\hline Totales & 664 & $100 \%$ & 616 & $100 \%$ \\
\hline
\end{tabular}

Tabla 5. Resultados generales de las variantes de /trc/ según género de los informantes en modalidad de lectura en voz alta

Alófonos de /țr/ en lectura según género de los informantes

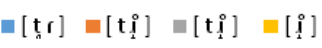

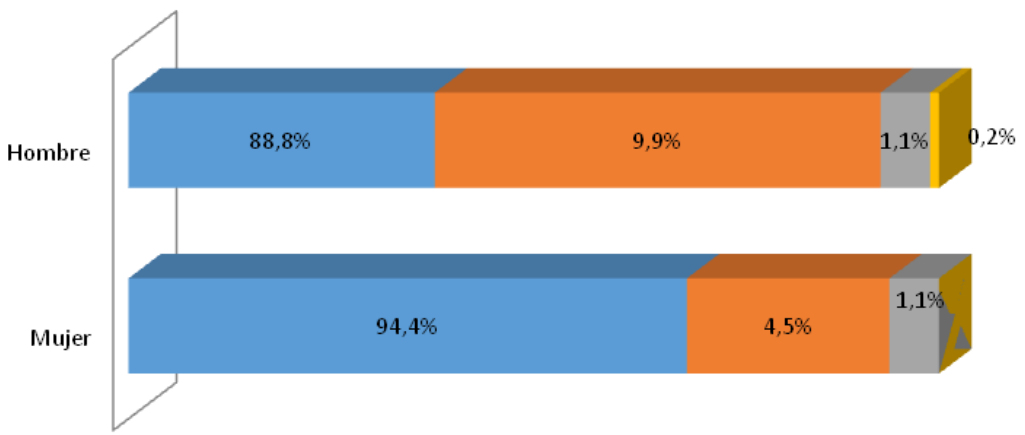

Figura 6. Gráfico de resultados generales de las variantes de /trr/ según género de los informantes en modalidad de lectura en voz alta

$\mathrm{Al}$ analizar el comportamiento alofónico del grupo consonántico /tr / a nivel nacional, según el género de los informantes en modalidad de lectura en voz alta, se observa en la Tabla 5 y en la Figura 6 que, en primer lugar, los hombres y las mujeres realizan con mayor frecuencia las variantes oclusiva seguida de vibrante simple [trc], la africada asibilada con fricción breve [t $[\mathrm{t}]$ y la variante africada asibilada con fricción larga [t] $\mathrm{t}]$, cuyos porcentajes de ocurrencia son: $88,8 \% ; 9,9 \%$ y $1,1 \%$ en el caso de los hombres; y $94,4 \%$; $4,5 \%$ y $1,1 \%$ en el caso de las mujeres. Por lo tanto, poseen comportamientos 
bastante similares en la jerarquía otorgada a los tres primeros alófonos, pero sus porcentajes son diferentes, ya que las mujeres realizan más oclusivas seguidas de vibrantes simples y menos africadas asibiladas con fricción breve en comparación con las realizaciones de los hombres. En segundo lugar, al analizar las realizaciones de la variante africada asibilada con fricción larga [t.I], se constata que tanto hombres como mujeres presentan siete realizaciones que equivalen a un $1,1 \%$. Además, los hombres presentan una ocurrencia de la variante fricativa [⿺辶⿸广巳i] $]$ con un $0,2 \%$ y las mujeres no presentan ninguna realización en toda la muestra. Por lo anterior, el comportamiento alofónico entre hombres y mujeres es similar en términos de la jerarquización de las variantes, pero difiere en términos de la relevancia porcentual que se le otorga a cada alófono, tendencias que se replican en modalidad de entrevista.

3.3. ANÁLISIS COMPARATIVO ENTRE LA MODALIDAD DE ENTREVISTA Y LECTURA

\begin{tabular}{|c|c|c|c|c|}
\hline & \multicolumn{2}{|c|}{ Entrevista } & \multicolumn{2}{|c|}{ Lectura } \\
\hline $\begin{array}{l}\text { Variante } \\
\text { fonética }\end{array}$ & $\begin{array}{l}\text { Número de } \\
\text { realizaciones }\end{array}$ & Porcentaje & $\begin{array}{l}\text { Número de } \\
\text { realizaciones }\end{array}$ & Porcentaje \\
\hline$[\underset{n}{\mathrm{t}} \mathrm{r}]$ & 1282 & $80,1 \%$ & 1174 & $91,7 \%$ \\
\hline$\left[\mathrm{t}^{1}\right]$ & 280 & $17,5 \%$ & 91 & $7,1 \%$ \\
\hline$\left[{ }^{\mathrm{t}} \mathrm{I}_{\mathrm{I}}^{\circ}\right]$ & 36 & $2,3 \%$ & 14 & $1,1 \%$ \\
\hline [ [̊] & 2 & $0,1 \%$ & 1 & $0,1 \%$ \\
\hline Totales & 1600 & $100 \%$ & 1280 & $100 \%$ \\
\hline
\end{tabular}

Tabla 6. Resultados generales de las variantes de $\underset{\Gamma}{\mathrm{tr}} /$ según modalidad discursiva 


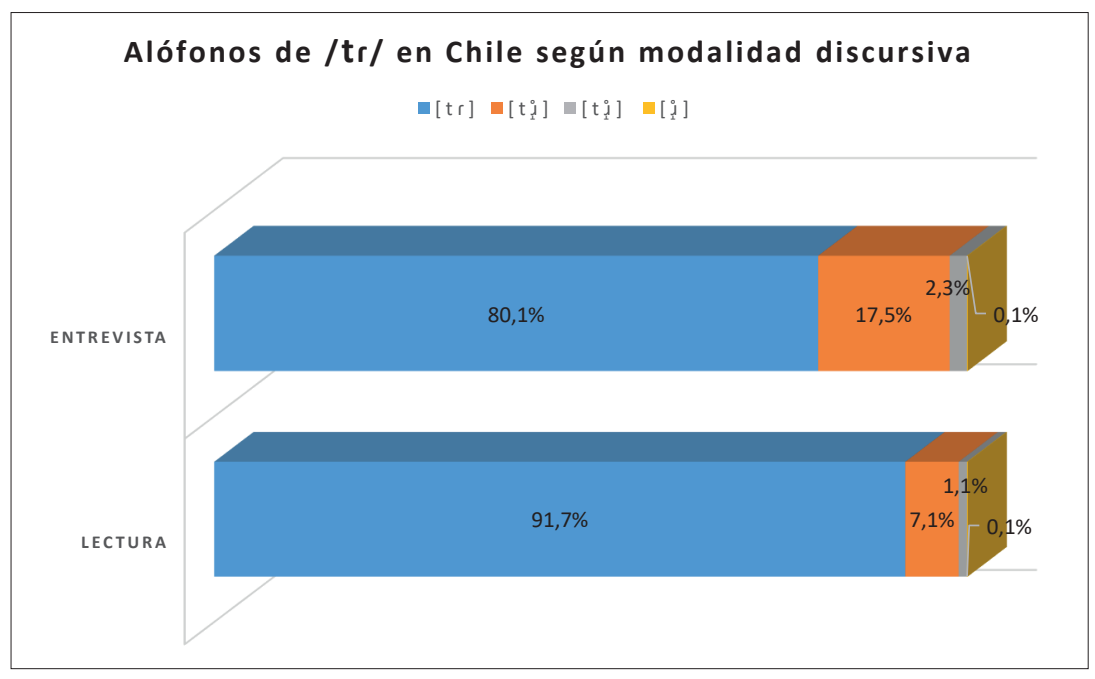

Figura 7. Gráfico de resultados generales de las variantes de $/ \mathrm{tr} /$ según modalidad discursiva

$\mathrm{Al}$ analizar el comportamiento alofónico del grupo consonántico /tr / a nivel nacional según modalidad discursiva se aprecia en la Tabla 6 y en la Figura 7 que, en primer lugar, tanto en modalidad de entrevista como de lectura en voz alta se presentan los cuatro alófonos en estudio con el mismo orden jerárquico y con distribuciones porcentuales similares, lo que permite afirmar cierta homogeneidad alofónica entre ambas modalidades discursivas. En segundo lugar, a pesar de lo mencionado anteriormente, se observan ciertas diferencias entre modalidades discursivas: en lectura se da un porcentaje mayor en el fono oclusivo seguido de vibrante simple [tr] (91,7\%) que en entrevista $(80,1 \%)$, por lo anterior, en el fono africado asibilado con fricción breve [t] en entrevista se da un mayor porcentaje $(17,5 \%)$ y en lectura un porcentaje menor $(7,1 \%)$, en el fono africado asibilado con fricción larga [t.J.] se constata un porcentaje mayor en entrevista $(2,3 \%)$ que en lectura $(1,1 \%)$;

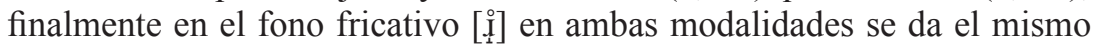
porcentaje $(0,1 \%)$. En tercer lugar, de acuerdo con la distribución porcentual en cada fono en estudio, se puede señalar que la modalidad discursiva de lectura en voz alta induce mayor control en la reproducción del discurso del informante, por lo que el porcentaje de la primera variante aumenta en un $11 \%$ con respecto a la modalidad de entrevista, la que propicia mayor espontaneidad en el habla. Por esto en la entrevista semiespontánea aumenta el porcentaje de la segunda variante en un $10 \%$ con respecto a la lectura. 


\subsection{EXPLORACIÓN ESTADÍSTICA DEL EFECTO DE VARIABLES CATEGÓRICAS EN LAS VARIANTES DE/T⿱ז口/}

Con la finalidad de complementar las observaciones cualitativas realizadas en las secciones anteriores, se llevará a cabo una serie de análisis cuantitativos para evaluar el efecto de las variables independientes zona geográfica, sexo y modalidad discursiva en la expresión de las variantes del grupo consonántico en estudio. En particular, se evaluará si existen asociaciones estadísticas entre las variables independientes antedichas y la variable dependiente variantes de $/ t r /$. En el caso de existir asociaciones, también se determinará qué variante de /trc/ es más o menos probable, y en qué magnitud, para cada nivel de cada variable categórica. Dicho de manera más sencilla, por ejemplo, se evaluará si existen diferencias en la proporción en la que hombres y mujeres usan variantes no estándares de /tr $/$, y cuánto más probable es que los hombres usen cierta variante por sobre otra, cuando se los compara con las mujeres.

\subsubsection{Efecto de la variable zona geográfica en las variantes de /tr /}

Una inspección preliminar de una tabla de contingencias que expresa las frecuencias de aparición de las cuatro variantes del grupo consonántico para las ocho ciudades en estudio reveló que existen varias celdas con una frecuencia de cero, y que más de un $20 \%$ de las celdas presenta frecuencias menores a cinco; ambas condiciones no permiten realizar análisis estadísticos como los que se requieren aquí, por lo que fue necesario colapsar niveles de algunas variables categóricas (Schwab 2002). En particular, se colapsaron los niveles de la variable dependiente variante de /tr $/ \mathrm{y}$ algunos niveles de la variable independiente ciudad. En el caso de la variable variante de / $t r /$, los cuatro niveles originales eran el fono "oclusivo seguido de vibrante simple [trc]", el fono "africado asibilado con fricción breve [th" "africado asibilado con fricción larga [t.J. fueron organizados de la siguiente manera: el nivel "variante 1" contiene

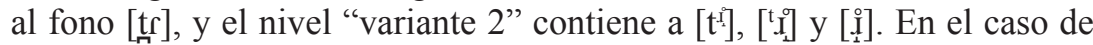
la variable ciudad, los ocho niveles fueron organizados por zona, de la siguiente manera: la zona "norte" contiene a los niveles "Iquique" y "La Serena", la zona "centro" contiene a "Santiago" y "Valparaíso", la zona "sur" a "Concepción" y "Temuco", y la zona "sur austral" a "Coyhaique" y "Punta Arenas". Esta reorganización para estas dos variables es la que se aplica para todos los subsecuentes análisis.

Como puede observarse en la Tabla 7, en general, para todas las zonas geográficas, existe una clara tendencia a que los participantes produzcan más variantes del primer tipo (la no marcada), con porcentajes cercanos al 
$80 \%$. Sin embargo, fuera de esta tendencia general, se observan porcentajes muy similares entre las zonas "norte" y "centro", pero diferencias entre estas dos zonas y las zonas "sur", y aún más claramente con la zona "sur austral", pues en las zonas "sur" y "sur austral", el porcentaje de aparición de variantes del tipo 2 aumenta. Con la finalidad de explorar una posible asociación entre las variables zona geográfica y variantes de /trr/, se realizó un análisis de Chi-cuadrado, el que reveló una asociación estadísticamente significativa entre las variables evaluadas $\left(\chi^{2}=24.493, \mathrm{df}=3, p<0.001 ; \mathrm{V}\right.$ de Cramer = 0.092); el tamaño de efecto, indicado en la V de Cramer, puede ser interpretado como pequeño (Kotrlik, Williams y Jabor 2011).

\begin{tabular}{|c|c|c|c|c|}
\hline Zona & Variante 1 & Variante 2 & Total fila & $\mathbf{( \% )}$ \\
\hline Norte & $\begin{array}{c}635 \\
(88,2 \%)\end{array}$ & $\begin{array}{c}85 \\
(11,8 \%)\end{array}$ & 720 & $(25 \%)$ \\
\hline Centro & $\begin{array}{c}636 \\
(88,3 \%)\end{array}$ & $\begin{array}{c}84 \\
(11,7 \%)\end{array}$ & 720 & $(25 \%)$ \\
\hline Sur & $\begin{array}{c}606 \\
(84,2 \%)\end{array}$ & $\begin{array}{c}114 \\
(15,8 \%)\end{array}$ & 720 & $(25 \%)$ \\
\hline Sur austral & $\begin{array}{c}579 \\
(80,4)\end{array}$ & $\begin{array}{c}141 \\
(19,6)\end{array}$ & 720 & $(25 \%)$ \\
\hline Total columna & $\begin{array}{c}2456 \\
(85,3 \%)\end{array}$ & $(14,7 \%)$ & 2880 & \\
\hline
\end{tabular}

Tabla 7. Tabla de contingencias para las variables variante fonética de /tr $r / \mathrm{y}$ zona geográfica. Se incluyen porcentajes por fila debajo de las frecuencias, entre

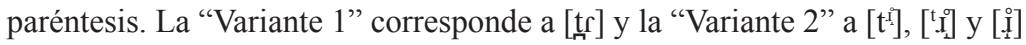

Para explorar más profundamente esta asociación, se llevó a cabo un análisis de Regresión Logística Multinomial, con variante de /trr/, como variable dependiente con dos niveles (nivel de referencia: "variante 1") y con zona geográfica como variable independiente con cuatro niveles (nivel de referencia: "centro"). Este tipo de análisis permite determinar si la variable independiente es capaz de predecir los niveles de la variable dependiente, y, si esto ocurre, qué manifestación de la variable dependiente es más probable. Los resultados del análisis, resumidos en la Tabla 8 , muestran que existen diferencias estadísticamente significativas en la manera en la que se usan las variantes de /tr $r$ entre las zonas "sur" y "centro", y entre las zonas "sur austral" y "centro", pero no entre las zonas "norte" y "centro", confirmando las tendencias observadas en la tabla de contingencias. En el caso de las diferencias entre la zona "sur" y la zona "centro", las razones de probabilidad muestran que es 1.42 veces más probable -o, lo que es lo 
mismo, un $42 \%$ más probable - que aparezca la "variante 2" que la "variante 1 " en la zona "sur" (cuando se la compara con la zona "centro"). En el caso de las diferencias entre las zonas "sur austral" y "centro", es un $84 \%$ más probable que aparezca la "variante 2" en lugar de la "variante 1" en la zona "sur austral", al ser comparada con la zona "centro".

\begin{tabular}{|c|c|c|c|c|c|c|}
\hline Comparaciones & $\begin{array}{c}\text { Estimados } \\
(\mathrm{ES})\end{array}$ & $\boldsymbol{p}$ & & $\begin{array}{c}\text { IC Bajo } \\
(2.5 \%)\end{array}$ & $\begin{array}{c}\text { Razones de } \\
\text { probabilidad }\end{array}$ & $\begin{array}{c}\text { IC Alto } \\
(97.5 \%)\end{array}$ \\
\hline Variante 2 vs. Variante 1 & & & & & & \\
\hline (Intercepto) & $-2.024(0.116)$ & $<0.001$ & $* * *$ & & & \\
\hline Zona Norte vs. Centro & $0.013(0.163)$ & $=0.082$ & & 0.105 & 1.013 & 0.165 \\
\hline Zona Sur vs. Centro & $0.353(0.154)$ & $<0.05$ & $*$ & 1.052 & 1.424 & 1.928 \\
\hline Zona Sur Austral vs. Centro & $0.611(0.149)$ & $<0.001$ & $* * *$ & 1.375 & 1.843 & 2.470 \\
\hline
\end{tabular}

Niveles de significatividad: $* * *<0.001, * *<0.01, *<0.05,<0.1$. Log-Likelihood $=-1191.4$. McFadden $\mathrm{R}^{2}$ : 0.0099977. Likelihood ratio test: $\chi^{2}=24.063, p<0.001$.

Tabla 8. Tabla resumen de los resultados de un análisis de Regresión Logística Multinomial con la variable variante de /tr/ como variable dependiente y zona geográfica como independiente. Se proveen los estimados, el error estándar de los mismos (ES), su valor de significatividad, razones de probabilidad e intervalos de confianza (IC) para las razones de probabilidad

\subsubsection{Efecto de la variable sexo en las variantes de /trr/}

Como puede observarse en la Tabla 9, en general, existe una tendencia a que tanto hombres como mujeres produzcan más variantes del primer tipo (la no marcada), en ambos casos con altos porcentajes. Sin embargo, el porcentaje de aparición de variantes del segundo tipo es mayor en los participantes de "sexo masculino" cuando se les compara con el "sexo femenino". Con la finalidad de explorar una posible asociación entre las variables sexo y variantes de /tr $/$, se realizó un análisis de Chi-cuadrado, el que reveló una asociación estadísticamente significativa $\left(\chi^{2}=62.23, \mathrm{df}=1, p<0.001\right.$; Coeficiente phi $=0.147)$; el tamaño de efecto, indicado por el Coeficiente phi, puede ser interpretado como pequeño (Kotrlik et al. 2011).

\begin{tabular}{|l|c|c|c|c|}
\hline Sexo & Variante 1 & Variante 2 & Total fila & $\mathbf{( \% )}$ \\
\hline Masculino & $\begin{array}{c}1107 \\
(79,9 \%)\end{array}$ & $\begin{array}{c}279 \\
(20,1 \%)\end{array}$ & 1386 & $(48,1 \%)$ \\
\hline Femenino & $\begin{array}{c}1349 \\
(90,3 \%)\end{array}$ & $\begin{array}{c}145 \\
(9,7 \%)\end{array}$ & 1494 & $(51,9 \%)$ \\
\hline
\end{tabular}




\begin{tabular}{|l|c|c|c|c|}
\hline Total columna & $\begin{array}{c}2456 \\
(85,3 \%)\end{array}$ & $\begin{array}{c}424 \\
(14,7 \%)\end{array}$ & 2880 & \\
\hline
\end{tabular}

Tabla 9. Tabla de contingencias para las variables variante fonética de /tr/y sexo. Se incluyen porcentajes por fila debajo de las frecuencias, entre paréntesis. La "Variante

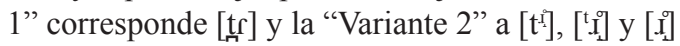

Para explorar más profundamente esta asociación, se llevó a cabo un análisis de Regresión Logística Multinomial, con variante de /tr/ como variable dependiente con dos niveles (nivel de referencia: "variante 1") y con sexo como variable independiente con dos niveles (nivel de referencia: "hombre $\rightarrow$ masculino"). Los resultados del análisis, resumidos en la Tabla 10, muestran que existen diferencias estadísticamente significativas en la manera en la que hombres y mujeres usan las variantes de $/ \mathrm{tr} /$. En particular, es un $57 \%$ menos probable que las mujeres usen la "variante 2" cuando se las compara con los hablantes de sexo masculino (o, lo que es lo mismo, es un $43 \%$ más probable que los hombres usen las variantes no estándar de $/ \mathrm{tr} /$ ).

\begin{tabular}{|c|c|c|c|c|c|c|}
\hline Comparaciones & $\begin{array}{c}\text { Estimados } \\
(\mathrm{ES})\end{array}$ & $p$ & & $\begin{array}{c}\text { IC } \\
\text { Bajo } \\
(2.5 \%)\end{array}$ & $\begin{array}{c}\text { Razones de } \\
\text { probabilidad }\end{array}$ & $\begin{array}{c}\text { IC Alto } \\
(97.5 \%)\end{array}$ \\
\hline Variante 2 vs. Variante 1 & & & & & & \\
\hline (Intercepto) & $-1.378(0.066)$ & $\begin{array}{c}< \\
0.001\end{array}$ & $* * *$ & & & 0.529 \\
\hline $\begin{array}{c}\text { Sexo masculino } \\
\text { vs. femenino }\end{array}$ & $-0.852(0.110)$ & $\begin{array}{c}< \\
0.001\end{array}$ & $* * *$ & 0.343 & 0.426 & 0.001 \\
\hline
\end{tabular}

Niveles de significatividad: $* * *<0.001, * *<0.01, *<0.05, .<0.1$. Log-Likelihood $=-1172$. McFadden $\mathrm{R}^{2}$ : 0.02614. Likelihood ratio test: $\chi^{2}=62.917, p<0.001$.

Tabla 10. Tabla resumen de los resultados de un análisis de Regresión Logística Multinomial con la variable variante de /tr/ como variable dependiente y sexo como independiente. Se proveen los estimados, el error estándar de los mismos (ES), su valor de significatividad, razones de probabilidad e intervalos de confianza (IC) para las razones de probabilidad

\subsubsection{Efecto de la variable modalidad discursiva en las variantes de /tr/}

Como puede observarse en la Tabla 11, en general existe una tendencia a que, en ambas modalidades discursivas, se utilicen más variantes no marcadas, sin embargo, el porcentaje de variantes marcadas es alrededor de un $10 \%$ mayor en la entrevista. Con la finalidad de explorar una posible asociación entre las variables modalidad discursiva y variantes de /tr $r$, se realizó un 
análisis de Chi-cuadrado, el que reveló una asociación estadísticamente significativa $\left(\chi^{2}=76.13, \mathrm{df}=1, p<0.001\right.$; Coeficiente $\left.\mathrm{phi}=0.163\right)$; el tamaño de efecto, indicado por el Coeficiente phi, puede ser interpretado como pequeño (Kotrlik et al. 2011).

\begin{tabular}{|l|c|c|c|c|}
\hline Modalidad discursiva & Variante 1 & Variante 2 & Total fila & $(\%)$ \\
\hline Entrevista & $\begin{array}{c}1282 \\
(80,1 \%)\end{array}$ & $\begin{array}{c}318 \\
(19,9 \%)\end{array}$ & 1600 & $(55,6 \%)$ \\
\hline Lectura & $\begin{array}{c}1174 \\
(91,7 \%)\end{array}$ & $\begin{array}{c}106 \\
(8,3 \%)\end{array}$ & 1280 & $(44,4 \%)$ \\
\hline Total columna & 2456 & $\begin{array}{c}424 \\
(14,7 \%)\end{array}$ & 2880 & \\
\hline
\end{tabular}

Tabla 11. Tabla de contingencias para las variables variante fonética de $/ t_{n} / \mathrm{y}$ modalidad discursiva. Se incluyen porcentajes por fila debajo de las frecuencias,

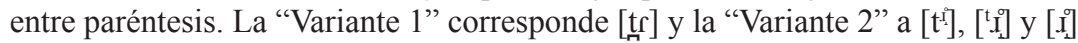

Para explorar más profundamente esta asociación, se llevó a cabo un análisis de Regresión Logística Multinomial, con variante de /trr/ como variable dependiente con dos niveles (nivel de referencia: "variante 1") y con modalidad discursiva como variable independiente con dos niveles (nivel de referencia: "entrevista"). Los resultados del análisis, resumidos en la Tabla 12, muestran que existen diferencias estadísticamente significativas en la manera en la que se expresan las variantes de $/ \mathrm{tr}_{\Gamma} /$ en las distintas modalidades discursivas. En particular, es un $64 \%$ menos probable que las variantes no estándares sean utilizadas en la "lectura", cuando se la compara con la "entrevista".

\begin{tabular}{|l|c|c|c|c|c|c|}
\hline Comparaciones & $\begin{array}{c}\text { Estimados } \\
(\mathrm{ES})\end{array}$ & $p$ & & $\begin{array}{c}\text { IC Bajo } \\
(2.5 \%)\end{array}$ & $\begin{array}{c}\text { Razones de } \\
\text { probabilidad }\end{array}$ & $\begin{array}{c}\text { IC Alto } \\
(97.5 \%)\end{array}$ \\
\hline Variante 2 vs. Variante 1 & & & & & & \\
\hline (Intercepto) & $-1.394(0.062)$ & $<0.001$ & $* * *$ & & & \\
\hline Entrevista vs. Lectura & $-1.010(0.119)$ & $<0.001$ & $* * *$ & 0.288 & 0.363 & 0.459 \\
\hline
\end{tabular}

Niveles de significatividad: $* * *<0.001, * *<0.01, *<0.05,<0.1$. Log-Likelihood $=-1163.4$. McFadden $\mathrm{R}^{2}$ : 0.033259. Likelihood ratio test: $\chi^{2}=80.05, p<0.001$.

Tabla 12. Tabla resumen de los resultados de un análisis de Regresión Logística Multinomial con la variable variante de /tr/como variable dependiente y modalidad discursiva como independiente. Se proveen los estimados, el error estándar de los mismos (ES), su valor de significatividad, razones de probabilidad e intervalos de confianza (IC) para las razones de probabilidad 


\section{CONCLUSIONES}

Sobre la base de los resultados expuestos en el apartado anterior, se puede concluir que la variante del grupo consonántico /trr/ más utilizada en el español no-estándar chileno, en situación de entrevista semiespontánea y de lectura en voz alta, es la oclusiva seguida de vibrante simple [trc] con porcentajes entre $80 \%$ y el $91 \%$; le sigue la variante africada asibilada con fricción breve [t] con porcentajes entre 7\% y el 17\%; luego está la africada asibilada con fricción larga [t.j] con porcentajes entre el 1 y el $2 \%$, finalmente se posiciona la variante fricativa $[.1$.$] con porcentajes menores$ del $0,1 \%$. Estos resultados no se ajustan a lo esperado para los hablantes de la muestra, ya que la bibliografía revisada indicaba que en el español no-estándar se encontrarían variantes africadas y fricativas con mayor prominencia porcentual.

Con respecto a la variable independiente zona geográfica, se puede afirmar que sí existe variación diatópica en las realizaciones de /tr / al comparar la zona norte y centro, con las zonas sur y sur-austral, ya que las primeras zonas geográficas mencionadas tienden a preferir las realizaciones no marcadas y en las dos últimas zonas mencionadas disminuyen estas realizaciones para darles paso a los fonos marcados, sin alterar la jerarquización de las variantes descrita en los párrafos precedentes. De acuerdo con las pruebas estadísticas realizadas, la incidencia de la zona geográfica en las realizaciones de /trr/ es estadísticamente significativa con un tamaño de efecto pequeño.

En relación con la variable independiente género de los informantes, se puede observar que las mujeres tienen más preferencia por las variantes no marcadas al ser comparadas con los hombres, esto sin alterar la jerarquización de las variantes descrita en la presente investigación. De acuerdo con las pruebas estadísticas realizadas, la incidencia del género de los informantes en las realizaciones de /trr/ es estadísticamente significativa con un tamaño de efecto pequeño. Estos resultados se encuentran en línea con lo que la literatura reporta sobre las diferencias sociolingüísticas entre hombres y mujeres, en el sentido de que los hombres suelen utilizar variantes de menor prestigio, aunque también más innovadoras, mientras que las mujeres prefieren variantes más prestigiosas (Silva-Corvalán 1989; Moreno 1990; Cepeda 1991; López 1993; Tassara 1993-1994).

En términos de la variable independiente modalidad discursiva, se puede sostener que los hablantes ejercen mayor control en la modalidad de lectura en voz alta, ya que aquí aumentan las realizaciones estándares, si se compara con la modalidad de entrevista semiespontánea donde estas disminuyen; lo anterior sin alterar la jerarquización de las variantes descritas 
en la presente investigación. De acuerdo con las pruebas estadísticas realizadas, la incidencia de la modalidad discursiva en las realizaciones de $/ t r /$ es estadísticamente significativa con un tamaño de efecto pequeño. Lo anterior puede ser explicado porque en la entrevista semiespontánea se da un estilo de habla informal, coloquial o casual, que tiene un ritmo más rápido, cambio de entonaciones, risas, digresiones y cambios espontáneos de temas, a diferencia de la lectura en voz alta que genera un estilo de habla cuidadoso y formal, porque el hablante presta mayor atención a su forma de hablar (Valdivieso, Merello y Candia, 1985, reeditada en el año 2007). En la misma línea, las variables, que condicionan el estilo de habla utilizado por el informante, son la autorregulación o el grado de atención que el hablante presta a su propio discurso, la relación de asimetría que existe entre el hablante y el investigador; y el tema del que se hable en la interacción comunicativa (Figueroa, et al., en evaluación).

Como una proyección del presente estudio se pueden analizar las diferencias y semejanzas en la distribución alofónica de /trc/ con el habla estándar en profesionales de Chile en modalidad de entrevista semiespontánea y de lectura en voz alta, trabajo desarrollado en el Proyecto Fondecyt 1120886 denominado "Descripción fonético-fonológica de un modelo de pronunciación enseñable del español culto de Chile", cuyo objetivo general fue identificar y describir el sistema fonético-fonológico del español de Chile que emplean los hablantes cultos, con el fin de proponer un modelo de pronunciación enseñable, y que es la contraparte de esta investigación.

\section{REFERENCIAS BIBLIOGRÁFICAS}

Alonso, Amado. 1953. Estudios lingüísticos. Temas hispanoamericanos. Madrid: Gredos.

Bobadilla, Félix y Gustavo Bobadilla. 1980-1981. El estudio de tres variables sociolingüísticas en Rancagua: problemas preliminares. Boletín de Filología Tomo XXXI: 721-241.

Boersma, P. y D. Weenink. 2018. Praat: doing phonetics by computer [programa computacional], versión 6.0.39, descargado el 3 de abril de 2018 de http://www.praat.org/

Canfield, Delos Lincoln. 1962. La pronunciación del español en América. Bogotá: Instituto Caro y Cuervo.

Cepeda, Gladys. 1991. Las consonantes de Valdivia. Valdivia: Imprenta América.

Contreras, Constantino. 1993. El castellano rural de Osorno, Chile. Estudios Filológicos 28: 123-135.

EsPINOSA, V. y M. ContARDO. 1992. La variación diastrática: un tipo de diferenciación interna considerado en el Atlas Lingüístico de la provincia de Parinacota. A.L.P.A. Revista de Lingüistica Teórica y Aplicada 30: 145-150. 
Figueroa, Mauricio. 2008. Prestigio de las variantes de /tr/ en la comuna de Concepción. Estudio sociolingüístico. Seminario para optar al grado de Licenciado en Educación, mención español. Universidad de Concepción.

Figueroa, Mauricio; Jaime Soto-Barba y Marco Ñanculeo. 2010. Los alófonos del grupo consonántico/tr/ en el castellano de Chile. Onomázein 22: 11- 42.

Figueroa, Mauricio; Gastón Salamanca y Marco Nanculeo. 2013. El eje oclusión-fricción en el sistema sociofónico del castellano chileno. Estudios de Fonética Experimental. XXII: 233-273.

Figueroa Candia, M. A.; D. A. García Rubio y G. F. Salamanca Gutiérrez (en evaluación). DiapixSp: Adaptación al español y aplicación piloto de una herramienta de elicitación de habla espontánea y colaborativa.

Fontanella, María. 1992. El español de América. Madrid: MAPFRE.

GAÍNZA, GASTÓN. (1976). El español de Costa Rica: breve consideración acerca de su estudio. Revista de Filología y Lingüística de la Universidad de Costa Rica 4: 79-83.

Hernández, Roberto; Carlos Fernández y María Baptista. 2014. Metodología de la investigación, $6^{\text {ta }}$ edición. México: McGRAW-HILL-Interamericana editores.

Kotrlik, J. W.; H. A. Williams, y M. K. JABOR. 2011. Reporting and Interpreting Effect Size in Quantitative Agricultural Education Research. Journal of Agricultural Education 52 (1): 132-142.

Lenz, Rodolfo. 1892. Chilenische Studien I. Alemania.

López, H. 1993. Sociolingüistica. Madrid: Gredos.

MenÉNDez Pidal, Ramón. 1962. Manual de gramática histórica española, $11^{\mathrm{a}}$ edic. Madrid: Espasa-Calpe.

Moreno Fernández, F. 1990. Metodología sociolingüística. Madrid: Gredos.

NaVArRo, Tomás. 1918. Manual de pronunciación española. $27^{\mathrm{a}}$ edic. Madrid: Consejo Superior de Investigaciones Científicas.

Oroz, Rodolfo. 1966. La lengua castellana en Chile. Santiago: Universidad de Chile, Facultad de Filosofía y Educación, Instituto de Filología.

Quilis, Antonio. 1999. Tratado de fonología y fonética españolas. Madrid: Gredos.

Rabanales, Ambrosio. 1992. El español de Chile: situación actual. Valladolid: Historia y presente del español de América. Junta de Castilla y León, Pabecal: 565-592.

2000. El español de Chile: presente y futuro. Onomázein 5: 135-141.

SALAS, AdALBERTO. 1996-1997. La lectura de noticias en la televisión chilena: modelo y norma en el fonetismo del castellano de Chile. Anuario de Lingüistica Hispánica Vol. XII-XIII Tomo $\mathrm{N}^{\circ}$ 2: 819-826.

Silva-Corvalán, C. 1989. Sociolingüistica. Teoría y análisis. Madrid: Alhambra.

Silva-Fuenzalida, Ismael. 1952-1953. Estudio fonológico del español de Chile. Boletín del Instituto de Filología de la Universidad de Chile (BFUCh) VII: 153-176.

SchwaB, J. A. 2002. Multinomial logistic regression: Basic relationships and complete problems [PowerPoint slides]. Retrieved from http://www.utexas.edu/courses/schwab/ sw388r7/SolvingProblems/

TASSARA, GILDA. 1993-1994. Valoración subjetiva de usos fonéticos alternativos en una muestra porteña. Nueva Revista del Pacífico: 8-39, 143-157.

VALDIVIESO, Humberto. 1978. El español culto y formal de Concepción. Revista de Lingüística Teórica y Aplicada 16: 125-133.

1988. Valoración subjetiva de los usos lingüísticos. Actas del Congreso Internacional de la Asociación de Lingüistica y Filología de América Latina 6. Universidad Nacional Autónoma de México, México D. F. 
Valdivieso, Humberto; Zoila Merello y Luis Candia. 2007. Fonética aplicada: diagnóstico y tratamiento de deficiencias en la lectura en voz alta. Revista de lingüistica teórica y aplicada 45: 37-58.

Véliz, María Orieta, Ángel Araya y Gustavo Rodríguez. 1977. Muestra del español hablado en las oficinas salitreras. Estudios Filológicos 12: 131-162.

Wagner, Claudio. 2004. El Atlas lingüístico y etnográfico de Chile por regiones (ALECH). Estudios Filológicos 39: 83-120.

WigdoRSKY, LEOPOLDO. 1978. Realización de algunos fonemas consonánticos en el castellano de Santiago. Informe preliminar. Revista de Lingüística Teórica y Aplicada 16: 53-60. 1983. Perfil fonosintáctico del castellano de Santiago de Chile, 1979. En J.J. Bergen y G.D. Bills (edits.). Spanish and Portuguese in social context. Georgetown University Press, pp. 76-87. 